\title{
Do Internal Mammary Nodes Matter?
}

\author{
Abigail S. Caudle, MD, MS and Benjamin D. Smith, MD \\ The University of Texas MD Anderson Cancer Center, Houston, TX
}

Surgeons continue to debate whether the removal of internal mammary (IM) nodes impacts outcomes in breast cancer patients. In a landmark randomized trial with 30 years of follow-up, Veronesi et al. ${ }^{1}$ established that routine dissection of this nodal region does not improve survival; however, the study was performed in a cohort of patients who did not receive adjuvant chemotherapy or radiation therapy. Does this still apply in the modern era of staging and treatment? Given improvements in assessing IM nodes with lymphoscintigraphy, coupled with more effective systemic therapy and more accurate radiation therapy, should we revisit this premise? Approximately $20-25 \%$ of patients have IM drainage on lymphoscintigraphy, and those with affected nodes have worse outcomes than nodenegative patients, which may in part be due to undertreatment when IM disease is not recognized. ${ }^{2-5}$ Knowing the pathologic state of this nodal region may help identify patients who would benefit from more aggressive therapy, as well as those who can safely avoid components of care.

van Loevezijn et al. ${ }^{5}$ attempt to address this in their current study. They identified patients from a prospectively maintained database at the Netherlands Cancer Institute who had IM drainage identified on preoperative lymphoscintigraphy. The power of this study is that removal of IM sentinel lymph nodes (SLNs) was routine practice, thus yielding novel insight into how this procedure impacted care. They report that in 336 patients with IM drainage seen, $287(85 \%)$ underwent successful removal of the IM SLNs. Outcomes were evaluated for three groups of patients: those with a pathologically positive IM SLN

(C) Society of Surgical Oncology 2019

First Received: 20 November 2018;

Published Online: 7 January 2019

A. S. Caudle, MD, MS

e-mail: ascaudle@mdanderson.org $(n=38)$; those with a pathologically negative IM SLN $(n=249)$; and those who did not have an IM node removed despite drainage seen $(n=49)$. Ten-year breast cancer-specific survival was $94 \%$ (95\% confidence interval [CI] 90-97\%) in patients with negative IM nodes, $78 \%$ (95\% CI 65-92\%) in patients with positive IM nodes, and $76 \%$ (95\% CI 63-89\%) in patients who did not have IM nodes removed.

Among the 287 patients with an IM SLN removed, 217 (76\%) had negative IM and axillary nodes, $32(11 \%)$ had positive axillary nodes and negative IM nodes, $18(6 \%)$ had solitary IM involvement, and $20(7 \%)$ patients had IM involvement with concurrent axillary disease. If we accept the premise that node-positive disease is a reasonable indication for cytotoxic chemotherapy, these findings indicate that integration of IM SLNs among patients with IM drainage on lymphoscintigraphy would result in a change in recommendation in favor of chemotherapy in up to $6 \%$ of patients. However, this fractional change is likely $<6 \%$ as some of these patients would receive chemotherapy based on primary tumor features, even if node-negative. This hypothesis is reflected in the study by van Loevezijn et al. as a change in systemic therapy recommendation was made in only seven patients, representing $2 \%$ of the cohort. Nevertheless, it is likely that the change in systemic therapy for this small fraction of patients could have favorably influenced outcomes. Other studies have shown similar results, including one from our own institution which found that adjuvant systemic therapy was impacted in 2 of 11 patients found to have a positive IM SLN. ${ }^{6}$ Madsen et al. ${ }^{3,7}$ found that the presence of IM nodal involvement did not impact prognosis independent of axillary metastases, with only $1 \%$ of patients receiving chemotherapy based on IM SLN status alone. While the number of patients where IM pathology impacts chemotherapy decisions is currently small, it may increase as efforts towards de-escalation of cytotoxic chemotherapy continue with integration of predictive molecular testing in 
the axillary node-positive setting. ${ }^{8,9}$ As we move to more personalized treatment based on the extent of nodal disease and not just the mere presence of any nodal disease, the information obtained from IM SLNs may become more critical.

The second question is whether the results of IM SLNs would influence radiation field planning. At present, indications for IM nodal irradiation are controversial. Historically, trials that defined the benefits of post-mastectomy radiation therapy for axillary node-positive patients included treatment of the IM nodal chain. ${ }^{10}$ However, utilization of IM nodal radiation among axillary node-positive patients has been relatively low in the US based on studies suggesting low rates of IM failure in the absence of IM radiation, coupled with concerns regarding higher lung and heart radiation exposure when the IM nodes are treated. ${ }^{11}$ Recently, long-term results of several studies that sought to clarify these issues have been reported. Unfortunately, none of these studies fully resolves the controversy. For example, a French trial included 1334 women with either axillary node-positive disease or central/medial N0 tumors, and randomized them to IM radiation or no IM radiation. With a median followup of 11 years, no differences in overall survival were noted. ${ }^{12}$ In contrast, a Danish population-based study compared outcomes among right-sided axillary node-positive patients who received IM radiation with left-sided axillary node-positive patients who did not receive IM radiation. With a median follow-up of 8 years, overall survival favored the right-sided patients who received IM radiation $(76 \%$ vs. $72 \% ; p=0.005){ }^{13}$

In light of the unclear and conflicting data regarding the risk-benefit ratio of IM nodal radiation, incorporation of IM SLNs could provide substantial benefit in guiding radiation decisions. For example, it would be very interesting to prospectively measure IM recurrence risk among axillary node-positive, IM SLN-negative patients treated without IM radiation. If IM recurrence risks were sufficiently low (preferably no higher than 1-2\%), then IM SLNs could emerge as a valuable strategy for facilitating a personalized approach to radiation treatment decisions, allowing many patients to forego the modest increase in morbidity associated with IM radiation treatment. In contrast, the identification of IM SLN-positive disease should absolutely result in the inclusion of the IM nodal chain in radiation treatment fields regardless of axillary disease status. Again, this would be helpful and would likely result in more patients who need IM nodal irradiation receiving it.

While the data showing poor outcomes when the IM nodes are not removed is intriguing, these data are not strong enough, in our opinion, to recommend routine removal of IM nodes based on this observation alone. The authors show that this group had similar outcomes to those with proven IM metastases. A closer look at these data suggests that there may have been other factors that could not be captured in this retrospective analysis. Thirteen percent of patients with sampled IM nodes had positive results on surgical pathology. Given that the baseline clinicopathologic features were similar to the group with IM nodes removed, one would expect a similar proportion with IM disease, which would translate into approximately six to seven patients. Over half of those would be predicted to have concurrent axillary disease and thus be receiving adjuvant chemotherapy and IM nodal radiation based on axillary pathology alone. In fact, this group without removal of IM nodes also had similar adjuvant therapy to those who had the nodes removed. Thus, the poor outcomes seen in this group cannot be from untreated IM nodal disease alone. Since it is impossible to retrospectively assess the surgical decision of whether to perform IM SLN or not, we can guess that these may have been patients at higher risk for distant disease, making surgeons less likely to perform a diagnostic procedure that carries increased operative risk. The authors also postulate that removing IM nodes may halt the spread of micrometasasis, but this seems unlikely and contrary to the early findings of Veronesi et al. that removal of IM nodes does not in itself convey a survival advantage. ${ }^{1}$

The authors conclude that IM SLNs should be removed in patients with tumors $>1.5 \mathrm{~cm}$ or aged $<70$ years. While these are reasonable guidelines, there may be an opportunity for other factors to impact this decision. The removal of IM SLNs is a diagnostic test with a complication rate of approximately $2-6 \% .^{5-7}$ As with any diagnostic test, the surgeon should consider how the results will impact adjuvant treatment planning before deciding whether to proceed with IM SLN dissection. First, will this change the decision about whether to receive chemotherapy? In patients in whom this answer is already known based on tumor biology, size, or known nodal involvement, the additional knowledge may not be beneficial. This decision algorithm may change in the next few years as the use of adjuvant systemic therapy evolves. The next question is whether IM node pathology might impact radiation decisions. Depending on institutional practice, a positive or negative IM SLN might be informative for radiation planning if IM coverage is not routinely employed.

In summary, removal of IM SLNs can provide critical information for a small subset of patients. Surgeons should continue to consider removal when the information it would glean may impact adjuvant decisions.

FUNDING This work has been supported by the Cancer Prevention and Research Institute of Texas (CPRIT; RP160670) [AC, BS], the 
National Cancer Institute (R01 CA207216) [BS], the Andrew Sabin Family Fellowship [BS], and a Cancer Center Support Grant from the National Institutes of Health (CA16672).

\section{REFERENCES}

1. Veronesi U, Marubini E, Mariani L, Valagussa P, Zucali R. The dissection of internal mammary nodes does not improve the survival of breast cancer patients. 30-year results of a randomised trial. Eur J Surg Oncol. 1999;35(9):1320-1325.

2. Kong A, Tereffe W, Hunt K, et al. Impact of internal mammary lymph node drainage identified by preoperative lymphoscintigraphy on outcomes in patients with stage I to III breast cancer. Cancer. 2012;118(24):6287-96.

3. Madsen E, Aalders K, van der Heidden-van der Loo M, et al (2015) Prognostic significance of tumor-positive internal mammary sentinel lymph nodes in breast cancer: a multicenter cohort study. Ann Surg Oncol. 22(13):4254-4262.

4. Amin M, Edge S, Greene F, Byrd D. AJCC staging manual. 8th ed. New York: Springer; 2016.

5. van Loevezijn A, Bartels S, van Duijnhoven F, et al. Internal mammary chain sentinel nodes in early stage breast cancer patients: towards selective removal. Ann Surg Oncol. https://doi. org/10.1245/s10434-018-7058-5.

6. Caudle A, Yi M, Hoffman K, et al. Impact of identification of internal mammary sentinel lymph node metastasis in breast cancer patients. Ann Surg Oncol. 2014;21(1):60-65.

7. Madsen E, Gobardhan P, Bongers V, et al. The impact on postsurgical treatment of sentinel lymph node biopsy of internal mammary lymph nodes in patients with breast cancer. Ann Surg Oncol. 2007;14(4):1486-1492.

8. Cardoso F, van'tVeer L, Bogarts J, et al. 70-gene signature as an aid to treatment decisions in early-stage breast cancer. $N$ Engl $J$ Med. 2016;375(8):717-729.

9. Ramsey S, Barlow W, Gonzalez-Angulo A, et al. Integrating comparative effectiveness design elements and endpoints into a phase III, randomized clinical trial (SWOG S1007) evaluating oncotypeDX-guided management for women with breast cancer involving lymph nodes. Contemp Clin Trials. 2013;34(1):1-9.

10. McGale P, Taylor C, et al. Effect of radiotherapy after mastectomy and axillary surgery on 10-year recurrence and 20-year breast cancer mortality: meta-analysis of individual patient data for 8135 women in 22 randomised trials. Lancet. 2014;383(9935):2127-2135.

11. Ceilley E, Jagsi R, Goldberg S, et al. Radiotherapy for invasive breast cancer in North America and Europe: results of a survey. Int J Radiat Oncol Biol Phys. 2005;61(2):365-373.

12. Hennequin C, Bossard N, Servagi-Vernat $S$, et al. Ten-year survival results of a randomized trial of irradiation of internal mammary nodes after mastectomy. Int J Radiat Oncol Biol Phys. 2013;86(5):860-866.

13. Thorsen L, Offersen B, Dan $\varnothing$ H, et al. DBCG-IMN: A Population-Based Cohort Study on the Effect of Internal Mammary Node Irradiation in Early Node-Positive Breast Cancer. J Clin Oncol. 2016;34(4):314-320.

Publisher's Note Springer Nature remains neutral with regard to jurisdictional claims in published maps and institutional affiliations. 